\title{
STEGANALYSIS OF BLOCK-DCT IMAGE STEGANOGRAPHY
}

\author{
Ying Wang and Pierre Moulin \\ University of Illinois at Urbana-Champaign \\ Beckman Institute, CSL \& ECE Dept. \\ 405 N. Mathews Ave., Urbana, IL 61801 USA
}

\begin{abstract}
Block-DCT based information embedding methods introduce distinctive non-stationarities into the stego-image. In particular, the difference between neighboring pixel intensities in one block and across two blocks have different distributions. A universal binary hypothesis test is then proposed to discriminate between stego-images and unmarked images.
\end{abstract}

\section{INTRODUCTION}

Steganography is a branch of information hiding [1]. An original image (cover-image) is modified by embedding secret information; the modified image is termed stego-image. The modification should be transparent in order to achieve perfectly secret communication. Here, perfect secrecy requires that in the stego-image, no detectable artifacts due to information embedding may be found to distinguish a stego-image from a legitimate cover-image. This implies that the stego-image should not deviate much from a given original cover-image according to a suitable distortion measure. Image steganographic techniques include least significant bit (LSB) embedding in spatial domain and discrete cosine transform (DCT) coefficients modification [1].

Conversely, the goal of steganalysis is to defeat steganography methods by identifying the presence of hidden information. This may be done using detection-theoretic methods if the distributions of the cover-image and stego-image are known to the steganalyzer [2], and various creative techniques otherwise [3].

In this paper, we focus on image steganalysis where information is hidden in $8 \times 8$ block-DCT coefficients. Because of the block structure of DCT embedding, pairs of neighboring pixels within an $8 \times 8$ block have different statistics from those across two $8 \times 8$ blocks. Two histograms of pixel differences are computed (one for each population), from which a Kolmogorov-Smirnov (K-S) binary hypothesis test is proposed to decide whether a given

This work was supported by NSF under grants CCR 00-81268 and CCR 02-08809. image is a stego-image, or a cover-image. A discussion of pros and cons of the K-S method is presented at the end of the paper.

\section{BLOCK-DCT DATA HIDING MODEL}

Consider a real-valued image (e.g. an 8-bit grayscale image) $u(m, n)$ with $m=1, \ldots, M$ and $n=1, \ldots, N$. We can divide the $M \times N$ image into $8 \times 8$ blocks and perform a two-dimensional (2-D) DCT on each of the $L=M N / 64$ blocks. For each block, the DCT coefficients are given by

$$
\tilde{u}(k, l)=\sum_{m=0}^{7} \sum_{n=0}^{7} c(k, m) u(m, n) c(l, n), \quad 0 \leq k, l \leq 7
$$

where $c(0, m)=\frac{1}{\sqrt{8}}$ and $c(k, m)=\sqrt{\frac{2}{8}} \cos \frac{\pi(2 m+1) k}{16}$, and $k=$ $1, \cdots, 7$.

The $8 \times 8$ block DCT generates 64 equal-size channels, which contain approximately independent data. The variance of $\tilde{u}(k, l)$ is denoted by $\sigma_{\tilde{u}}^{2}(k, l)$.

The DCT coefficients for the stego-image are constructed using a modified spread-spectrum technique:

$$
\tilde{u}^{\prime}(k, l)=\tilde{v}(k, l)+\tilde{z}(k, l),
$$

where $\tilde{v}(k, l)=\tilde{a}_{k, l} \tilde{u}(k, l), \tilde{a}_{k, l}=1-\tilde{D}_{k, l} / 2 \sigma_{\tilde{u}}^{2}(k, l)$ and $\tilde{z}(k, l) \sim \mathcal{N}\left(0, \frac{1+\tilde{a}_{k, l}}{2} \tilde{D}_{k, l}\right)$. The per-coefficient distortions $\tilde{D}_{k, l}=E\left|\tilde{u}^{\prime}(k, l)-\tilde{u}(k, l)\right|^{2}$ are allocated according to minimum square error (MSE) constraints or to a just noticeable difference (JND) threshold. We select $\tilde{D}_{k, l}=d_{e} \sigma_{\tilde{u}}^{2}(k, l)$, where $d_{e}$ is termed the normalized embedding distortion. The values of $\tilde{a}_{k, l}$ and $\sigma_{\tilde{z}}^{2}(k, l)$ are such that $\sigma_{\tilde{u}^{\prime}}^{2}(k, l)=$ $\sigma_{\tilde{u}}^{2}(k, l)$, which is required for Gaussian stego-systems to satisfy the condition that the Kullback-Leibler (K-L) distance $D\left(p_{\tilde{u}(k, l)}|| p_{\tilde{u}^{\prime}(k, l)}\right)$ is zero.

Transforming $\tilde{u}^{\prime}(k, l)$ back to the spatial domain, we obtain the stego-image as

$$
u^{\prime}(m, n)=v(m, n)+z(m, n)
$$




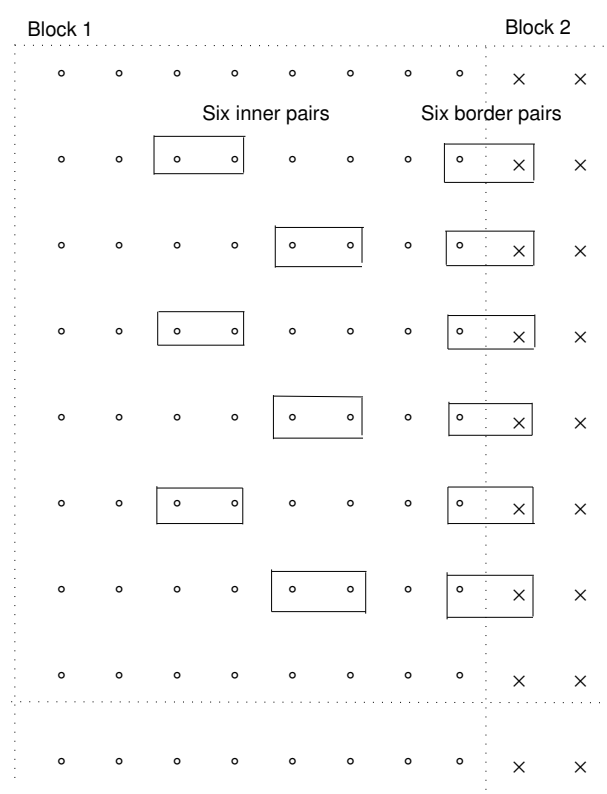

Fig. 1. Selected inner and border pairs

where

$$
\left[\begin{array}{c}
u^{\prime} \\
v \\
z
\end{array}\right](m, n)=\sum_{k=0}^{7} \sum_{l=0}^{7} c(k, m)\left[\begin{array}{c}
\tilde{u}^{\prime} \\
\tilde{v} \\
\tilde{z}
\end{array}\right](k, l) c(l, n) .
$$

Hence, the stego-image consists two parts: $v(m, n)$ and $z(m, n)$. The former part is a function of the cover-image $u(m, n)$ and the masking $\tilde{a}(k, l)$. The second part is a hidden noise-like pattern, independent of the cover-image.

\section{STATISTICS OF THE VALUE DIFFERENCE BETWEEN NEIGHBORING PIXELS}

We define the value difference of a horizontally neighboring pair as $d_{m, n}=u_{m, n}-u_{m, n-1}{ }^{1}$ for the cover-image and $d_{m, n}^{\prime}=u_{m, n}^{\prime}-u_{m, n-1}^{\prime}$ for the stego-image.

Assume that the cover-image is a 2-D stationary process with zero mean and correlation function $r_{u}(k, l)$. Then $d_{m, n}$ is also a stationary process with zero mean and correlation function

$$
\begin{aligned}
r_{d}(k, l) & =E\left[d_{m, n} d_{m-k, n-l}\right] \\
& =2 r_{u}(k, l)-r_{u}(k, l+1)-r_{u}(k, l-1) .
\end{aligned}
$$

We select pairs within a block and across two blocks as shown in Fig. 1. Since $d_{m, n}$ is a stationary process, naturally the probability density functions (pdf) $p_{0}$ for the inner pairs and $p_{1}$ for the border pairs are the same. Fig. $2 \mathrm{a}$

\footnotetext{
${ }^{1}$ For the purpose of compactness, we will use $d_{m, n}$ or $d\left(\begin{array}{c}m \\ n\end{array}\right)$ instead of $d(m, n)$ when no confusion occurs.
}
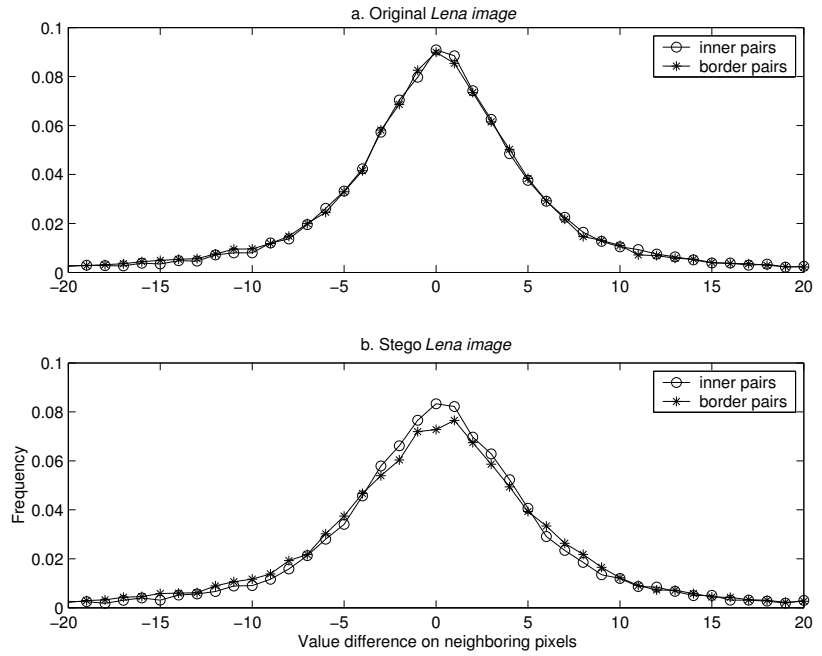

Fig. 2. Histograms for value difference from inner and border pairs based on a. original image Lena and b. stegoimage Lena. The DCT coefficients $\tilde{u}(k, l)$ with variance between 10 and 500 of original Lena were marked according to (2) and $d_{e}=0.05$.

compares the histograms of these two pdfs for image Lena; they are indeed remarkably similar.

To calculate $r_{d^{\prime}}(m, n ; m-k, n-l)=E\left[d_{m, n}^{\prime} d_{m-k, n-l}^{\prime}\right]$ for a stego-image, we need to look at $r_{z}(m, n ; m-k, n-l)$ and $r_{v}(m, n ; m-k, n-l)$ first.

We rewrite $(m, n)$ as $\left(m_{0}+m_{0}^{\prime}, n_{0}+n_{0}^{\prime}\right)$, where $\left(m_{0}, n_{0}\right)$ is the global index for the most upper-left pixel of the block to which $(m, n)$ belongs, and $\left(m_{0}^{\prime}, n_{0}^{\prime}\right)$ are the offset indexes of $(m, n)$ in that block. Similarly, we rewrite $(m-$ $k, n-l)$ as $\left(m_{1}+m_{1}^{\prime}, n_{1}+n_{1}^{\prime}\right)$. From (4), it is easy to check that

$r_{z}\left(\begin{array}{c}m_{0}+m_{0}^{\prime} \\ n_{0}+n_{0}^{\prime}\end{array} ; \begin{array}{c}m_{0}+m_{1}^{\prime} \\ n_{0}+n_{1}^{\prime}\end{array}\right)=\sum_{i, j=0}^{7} c_{i, m_{0}^{\prime}} c_{j, n_{0}^{\prime}} c_{i, m_{1}^{\prime}} c_{j, n_{1}^{\prime}} \sigma_{\tilde{z}}^{2}(i, j)$

for two pixels in the same block, and zero otherwise since $\tilde{z}(k, l)$ 's are independent. Hence, $z(m, n)$ is not a stationary process. Usually, $v(m, n)$ is also not stationary because of the nonuniform masking $\tilde{a}(k, l)$. Therefore $u^{\prime}(m, n)$ is nonstationary too.

Because of the stationarity of $u(m, n)$, we have $\sigma_{d}^{2}(m, n)$ $=2\left[\sigma_{u}^{2}-r_{u}(1)\right]$. However, for $\sigma_{d^{\prime}}^{2}(m, n)$, we have to distinguish between two cases: $d_{0}^{\prime}(m, n)$ from inner pairs and $d_{1}^{\prime}(m, n)$ from border pairs.

For $d_{0}^{\prime}(m, n)$ from pairs within a block, the variance is

$$
\begin{aligned}
& \sigma_{d_{0}^{\prime}}^{2}(m, n)=\sigma_{v}^{2}(m, n)+\sigma_{v}^{2}(m, n-1)-2 r_{v}(\underset{n}{m} ; \underset{n-1}{m,}) \\
& +\sigma_{z}^{2}(m, n)+\sigma_{z}^{2}(m, n-1)-2 r_{z}\left(\begin{array}{c}
m \\
n
\end{array} ;{ }_{n-1}^{m,}\right),
\end{aligned}
$$

where $(n \bmod 8) \neq 0$. While for $d_{1}^{\prime}(m, n)$ from pairs across 
two blocks, the variance is

$$
\begin{aligned}
\sigma_{d_{1}^{\prime}}^{2}(m, n) & \left.=\sigma_{v}^{2}(m, n)+\sigma_{v}^{2}(m, n-1)-2 r_{v} \underset{n \rightarrow m}{\underset{n}{m} ; m, 1}\right) \\
& +\sigma_{z}^{2}(m, n)+\sigma_{z}^{2}(m, n-1),
\end{aligned}
$$

where $(n \bmod 8)=0$. Both $\sigma_{d_{0}^{\prime}}^{2}(m, n)$ and $\sigma_{d_{1}^{\prime}}^{2}(m, n)$ are periodic with respect to the $8 \times 8$ block.

Comparing (7) and (8), there are two major differences between $\sigma_{d_{0}^{\prime}}^{2}(m, n)$ and $\sigma_{d_{1}^{\prime}}^{2}(m, n)$. First, because of the independence of $z(m, n)$ from block to block, the term $-2 r_{z}(m, n ; m, n-1)$ in (7) disappears from (8). Second, although the expression $-2 r_{v}(m, n ; m, n-1)$ in (7) is the same as in (8), a pair of $v(m, n)$ and $v(m, n-1)$ in the same block only involves all the pixels within that block, while a pair across two blocks involves two blocks of pixels, which are more remotely related. If $v(m, n)$ 's or $z(m, n)$ 's are positively correlated, which is the usual case, then the variances from (7) tend to be smaller than those from (8).

Therefore, although both the pdf $p_{0}^{\prime}$ for $d_{0}^{\prime}(m, n)$ from inner pairs and the pdf $p_{1}^{\prime}$ for $d_{1}^{\prime}(m, n)$ from border pairs have zero mean, they differ on variances: $\bar{\sigma}_{d_{0}^{\prime}}^{2}$ and $\bar{\sigma}_{d_{1}^{\prime}}^{2}$. The variances are averaged over those selected pairs as shown in Fig. 1 and their difference will depend on the normalized embedding distortion $d_{e}$. As an example, Fig. $2 \mathrm{~b}$ shows the histograms of these two pdfs for image Lena with hidden information. Apparently, this difference between $d_{0}^{\prime}(m, n)$ and $d_{1}^{\prime}(m, n)$ reveals the trace of a stego-image, which differs from a legitimate cover-image.

\section{BINARY HYPOTHESIS TESTING PROBLEM}

If the detector knew $p_{0}$ or $p_{0}^{\prime}$ and $p_{1}^{\prime}$, the detection of stegoimages would be a simple hypothesis testing problem. The likelihood ratio test (LRT) could then be applied to obtain an appropriate decision. In practice, however, the pdf's are not known exactly. The common case would be that the detector observes two sets of finite samples from unknown pdfs $p_{0}^{\prime}$ and $p_{1}^{\prime}$, then tries to infer whether these two pdfs are identical or not, i.e. to decide that the observed image is an unmarked image or a stego-image. This suggests using a two-sample goodness-of-fit test from the nonparametric statistics literature. Either the chi-square $\left(\chi^{2}\right)$ test or the Kolmogorov-Smirnov (K-S) test can be used to form decision rules. Here we choose to use the K-S test because the $\chi^{2}$ test is affected by the number of classes and their widths, which is not easy to control [4].

The performance of above tests has been well studied when the observed samples are independent. Observing (6) and (8), we can reasonably approximate the $d_{0}^{\prime}(m, n)$ 's and the $d_{1}^{\prime}(m, n)$ 's as independent because of the bell shape of $r_{u}(k, l)$ and the orthogonality of the DCT basis functions, which result in low correlations for $d_{0}^{\prime}(m, n)$ 's or $d_{1}^{\prime}(m, n)$ 's at different locations.
For samples drawn independently from two populations, $\left\{d_{0}^{\prime}\right\}$ and $\left\{d_{1}^{\prime}\right\}$, with cumulative probability distributions $F_{0}(x)$ and $F_{1}(x)$, the hypotheses are [4]

$$
\left\{\begin{array}{l}
H_{0}: F_{0}=F_{1} \\
H_{1}: F_{0} \neq F_{1}
\end{array}\right.
$$

Define the two empirical distribution functions from the observations on two populations with respective sizes $M$ and $N$ as

$$
\left\{\begin{array}{l}
S_{0}(x)=\#\left\{d_{0}^{\prime}(m, n) \leq x\right\} / M \\
S_{1}(x)=\#\left\{d_{1}^{\prime}(m, n) \leq x\right\} / N
\end{array}\right.
$$

The two-sample K-S goodness-of-fit test statistic is defined as the $L_{\infty}$ distance between $S_{0}(x)$ and $S_{1}(x)$, or

$$
D_{M, N}=\sup _{x}\left|S_{0}(x)-S_{1}(x)\right|
$$

Under $H_{0}$, for any continuous probability distribution $F(x)$, $\sqrt{\frac{M N}{M+N}} D_{M, N}$ weakly converges to $\sup _{0 \leq t \leq 1}|X(t)-t X(1)|$, where $X(t)$ is normalized Brownian motion [5]. The asymptotic distribution is

$\lim _{M, N \rightarrow \infty} P_{0}\left(\sqrt{\frac{M N}{M+N}} D_{M, N} \leq d\right)=1-2 \sum_{k=1}^{\infty}(-1)^{k-1} e^{-2 k^{2} d^{2}}$.

Let the right hand side of (12) be $1-\alpha$, where $\alpha$ is the maximum allowed error probability of false alarm $P_{F A}$. Then we can calculate the corresponding threshold $D_{M, N, \alpha}$. The decision rule with $P_{F A} \leq \alpha$ is

$$
\delta_{D}\left(\left\{d_{0}^{\prime}\right\},\left\{d_{1}^{\prime}\right\}\right)=\left\{\begin{array}{ccc}
1 & > \\
0 \text { or } 1 & D_{M, N} & =D_{M, N, \alpha} \\
0 & & <
\end{array}\right.
$$

Actually, the distributions for $\left\{d_{0}^{\prime}\right\}$ and $\left\{d_{1}^{\prime}\right\}$ are discrete. When used for discrete distributions, the K-S test tends to be conservative on rejecting $H_{0}$ [4], i.e. the actual $D_{M, N, \alpha}$ should be a smaller value.

The behavior of $D_{M, N}$ under various embedding strengths is shown in Fig. 3 for the images Lena, Jet and Baboon. The result indicates that with the same embedding ratio $d_{e}$ (a few percent), the adulterated versions of smooth images are more likely to be detected than those of images with noiselike textures. That is because for images such as Baboon, the variances of $\left\{d_{0}^{\prime}\right\}$ and $\left\{d_{1}^{\prime}\right\}$ are so large that the difference between cover- and stego-images is relatively small and cannot be identified easily. This in turn suggests that the better candidates for cover-images are noise-like images, and that block-DCT steganography is not suitable for smooth images. 


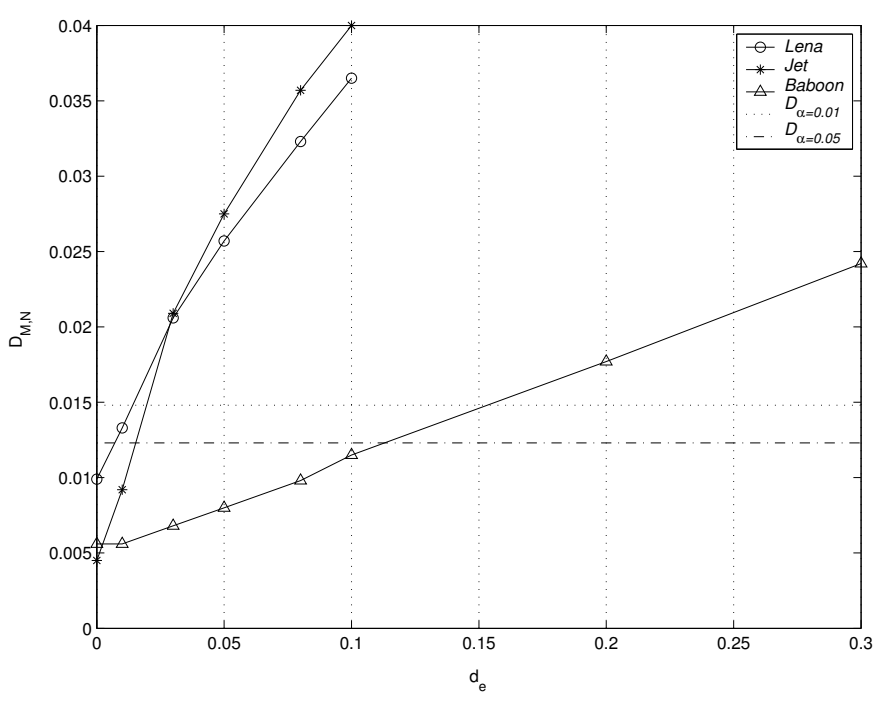

Fig. 3. Observed $D_{M, N}$ vs. normalized embedding distortion $d_{e}$, where $M=24576$ and $N=24192$. The DCT coefficients $\tilde{u}(k, l)$ with variance between 10 and 500 of original images were marked according to (2).

\section{DISCUSSION}

The non-stationarity introduced by block-DCT steganography reveals the existence of hidden information. The difference between the distributions for pixel pairs from one block and across blocks provides a measure for the detection of stego-images. Similar ideas can be used to detect discrete wavelet transform (DWT) steganography. Because of the upsampling by factor 2 , a stationary process in one subband will normally result in a non-stationary process in the spatial domain after the IDWT. For example, assume that an i.i.d noise sequence is embedded in the highpass subband of a stationary 1-D host signal. Then the stego-signal is a combination of two stationary sequences: one consisting of the even samples and the other of the odd samples. Doing the K-S test on these two populations, we can in principle tell if there is a stationary process embedded into the DWT domain.

A K-S test is used in this paper to form the decision rule for the nonparametric hypothesis test on whether two populations are from different distributions. The $\chi^{2}$ test can replace the K-S test and give similar detection performance. From the derivation of the $\chi^{2}$ goodness-of-fit test on a single population [4], we can see the $\chi^{2}$ test is actually related to a generalized likelihood ratio test (GLRT). Suppose $y$ belongs to a discrete alphabet $\mathcal{Y}$. The null hypothesis specifies the probability mass function (pmf) $\left\{p_{0}(y)\right\}$ completely and the alternative hypothesis specifies $\left\{p_{1}(y)\right\}$. Then the $\log$ likelihood ratio for $N$ observations is

$$
L\left(y^{N}\right)=\sum_{k=1}^{N} \log \frac{p_{1}\left(y_{k}\right)}{p_{0}\left(y_{k}\right)}=N \sum_{i \in \mathcal{Y}} \hat{p}_{i} \log \frac{p_{1, i}}{p_{0, i}}
$$

where $\hat{p}_{i}=\#\left\{k: y_{k}=i\right\} / N, i \in \mathcal{Y}$ is the empirical pmf. In the $\chi^{2}$ test, $\hat{p}_{i}$, which is the maximum-likelihood estimate (MLE) of the pmf of the observed sequence, is used to replace the unknown alternative distribution $p_{1, i}$ :

$$
L_{\chi^{2}}\left(y^{N}\right)=N \sum_{i \in \mathcal{Y}} \hat{p}_{i} \log \frac{\hat{p}_{i}}{p_{0, i}}
$$

Therefore, for the LRT (14), the expectation of $L\left(y^{N}\right)$ is $-N D\left(p_{0} \| p_{1}\right)<0$ under $H_{0}$ and $N D\left(p_{1} \| p_{0}\right)>0$ under $H_{1}$, respectively. However, for the $\chi^{2}$ test, $L_{\chi^{2}}\left(y^{N}\right)$ is $N D\left(\hat{p}_{0} \| p_{0}\right) \geq 0$ under $H_{0}$ and $N D\left(\hat{p}_{1} \| p_{0}\right)$ (which is near $N D\left(p_{1} \| p_{0}\right)$ if the MLE is close to the actual pmf) under $H_{1}$, respectively. The $\chi^{2}$ test has a smaller separation between the expectations of test statistics under two hypotheses. The above reasoning suggests that the LRT can do better than the $\chi^{2}$ test in [7], where a good estimate of unknown $p_{1, i}$ can be found by exploiting the smoothness of image pixel histograms. Back to our problem, we have two unknown pmfs: we have to find an estimate for $p_{0, i}$ too. Since we don't assume any priors for the two populations, nonparametric universal tests such as $\chi^{2}$ and K-S tests fit well here. However, this universality may imply a performance cost for smooth pdf's.

Our proposed steganalysis method works better for smooth images than for noise-like images. This suggests that our method could be further improved by categorizing images into different classes according to their smoothness, and making the decision rule adaptive to the nature of images.

\section{REFERENCES}

[1] N. F. Johnson and S. Katzenbeisser, "A survey of steganographic techniques", in S. Katzenbeisser and F. Peticolas (Eds.): Information Hiding, pp.43-78. Artech House, Norwood, MA, 2000.

[2] C. Cachin, "An information-theoretic model for steganography", in D. Aucsmith (Ed.): Information Hiding, 2nd International Workshop, vol. 1525 of Lectures Notes in Computer Science, pp. 306-318. Springer, 1998.

[3] J. Fridrich and M. Goljan, "Practical steganalysis of digital images-state of the art", Proc. SPIE Photonics West, Vol. 4675, pp. 1-13, San Jose, California, Jan. 2002.

[4] J. D. Gibbons and S. Chakraborti, Nonparametric statistical inference, Marcel Dekker, New York, 1992.

[5] L. Breiman, Probability, SIAM, Philadelphia, 1992.

[6] M. Barni, F. Bartolini and A. Piva, "Improved wavelet-based watermarking through pixel wise masking", IEEE Trans. on Image Processing, vol. 10, no. 5, pp. 783-791, May 2001.

[7] O. Dabeer, K. Sullivan, U. Madhow, S. Chandrasekharan, and B. S. Manjunath, "Detection of hiding in the least significant bit”, Proc. CISS, The Johns Hopkins University, Mar. 2003. 